OPEN ACCESS

Edited by:

Yvonne Höller,

University of Akureyri, Iceland

Reviewed by:

Fedele Dono,

University of Studies G. d'Annunzio

Chieti and Pescara, Italy

Patrizia Pulitano,

Sapienza University of Rome, Italy

${ }^{*}$ Correspondence:

Kai Zhang

coolzhangkai@163.com

Zhen Zhou

zhouzhen7681@126.com

TORCID:

Meidan Zhao

orcid.org/0000-0001-9055-4805

Kai Zhang

orcid.org/0000-0002-7248-1451

Zhen Zhou

orcid.org/0000-0002-8370-8981

¥These authors have contributed equally to this work

Specialty section:

This article was submitted to Epilepsy,

a section of the journal

Frontiers in Neurology

Received: 22 May 2021 Accepted: 02 August 2021

Published: 26 August 2021

Citation:

Zhang $Y$, Zhao $M$, Zhang $B$, Zhang $K$ and Zhou Z (2021) Acupuncture as an Adjunctive Treatment for Post-stroke Epilepsy: Protocol for a Randomized

Controlled Trial.

Front. Neurol. 12:711390

doi: 10.3389/fneur.2021.711390

\section{Acupuncture as an Adjunctive Treatment for Post-stroke Epilepsy: Protocol for a Randomized Controlled Trial}

\author{
Yang Zhang ${ }^{1 \neq}$, Meidan Zhao ${ }^{2 \neq \ddagger}$, Baozhen Zhang ${ }^{3 \ddagger}$, Kai Zhang ${ }^{4 \star t}$ and Zhen Zhou ${ }^{5 * t}$ \\ ${ }^{1}$ Rehabilitation Department, Tianjin Nankai Hospital, Tianjin, China, ${ }^{2}$ Department of Acu-moxibustion and Tuina, Tianjin \\ University of Traditional Chinese Medicine, Tianjin, China, ${ }^{3}$ Department of Health Management, Tianjin Rehabilitation and \\ Convalescence Center of PLA, Tianjin, China, ${ }^{4}$ Department of Acupuncture and Moxibustion, Tianjin Gong An Hospital, \\ Tianjin, China, ${ }^{5}$ Department of Acupuncture and Moxibustion for Encephalopathy, The Second Hospital Affiliated to Tianjin \\ University of Traditional Chinese Medicine, Tianjin, China
}

Background: Acupuncture has been clinically used to treat epilepsy after stroke. However, most of the current clinical studies are observational studies, and there are few well-designed randomized controlled trials (RCTs). Hence, we designed a multicentre RCT to assess the advantages and efficacy of acupuncture for post-stroke epilepsy (PSE).

Methods/Design: This is a two-arm, parallel, participants-blinded and assessor-blinded and multicentre RCT. A total of 120 patients with PSE aged from 18 to 75 years will be randomly assigned to two groups (routine treatment plus acupuncture group and routine treatment plus sham acupuncture group) at a 1:1 ratio. The participants will perform acupuncture or sham acupuncture treatment three times a week and be ongoing 8-week treatment. The primary endpoint is the proportion of seizure-free patients. A safety profile will be established. We will record adverse events for the safety evaluation.

Discussion: The study will provide high-quality clinical evidence on the effectiveness and safety of acupuncture for treating patients with epilepsy after stroke.

\section{Clinical Trial Registration: Chinese Clinical Trial Registry, identifier: ChiCTR2100046114.}

Keywords: acupuncture, post-stroke epilepsy, randomized controlled trial, study protocol, neurology

\section{INTRODUCTION}

Stroke is a common cause of epilepsy in adults, causing about $10 \%$ of all epilepsy and $55 \%$ of newly diagnosed seizures in older adults (1). Although recent advances in the diagnosis and treatment of acute stroke have increased patient's life expectancy, the incidence of Post-stroke epilepsy (PSE) is also on the rise (1). PSE is defined as unprovoked epileptic seizures at least 1 week after stroke and occurs in at least $4-6 \%$ of the stroke patients (2). PSE is one of the major complications after stroke. A meta-analysis found that younger age, hemorrhagic component, and cortical involvement at stroke onset were associated with a higher risk of PSE (3). PSE may be associated with increased mortality and unfavorable outcomes. In addition, there is a high risk of seizure recurrence following an unprovoked seizure after a stroke. Although the European Stroke 
Organization guidelines recommend that treatment with antiepileptic drugs (AEDs) should be considered after one unprovoked seizure (4), there is currently limited evidence on the efficacy and safety of AEDs for PSE $(1,4,5)$. The management of PSE is also challenged by several factors, such as the changes in pharmacodynamics due to older age, co-morbidities, and co-medications in stroke survivors. Older people, in particular, may be more susceptible to side effects from antiseizure medications (5).

As an essential part of traditional Chinese medicine (TCM), acupuncture has a history of more than 3,000 years in China and spread to Europe and American between the 16th and the 19th century. As one of the oldest medical approaches globally, acupuncture is a complementary medicine modality that involves inserting needles into specific parts of the body and then stimulating them by manual operation, electric pulses, or heat (6). Doctors and scientists have struggled to assess the actual effects of acupuncture and the underlying biological and physiological mechanisms of acupuncture. Clinical trials in different countries have shown that acupuncture may have beneficial effects on various diseases, including osteoarthritis, stroke, gastrointestinal disorders, cancer pain, and so on (711). Also, during the COVID-19 epidemic, TCM, including acupuncture, was widely used in treating COVID-19 patients, and acupuncture may have a positive effect on patients (12, 13). There has already evidence that acupuncture is feasible for treating movement disorders (14), as routine treatment has significant limitations. As a personalized treatment, acupuncture offers the potential to treat refractory epilepsy $(15,16)$. The imbalance between the sympathetic and parasympathetic activities may cause different diseases, and ultimately damage different brain regions. Researchers have found that acupuncture can not only activate the above-mentioned brain regions, but also relieving the autonomic nervous response by regulating the relevant adaptive neurotransmitters in distinct brain areas (16). Some experiments have investigated the mechanisms of action underlying acupuncture in treating epilepsy. An animal study shows that acupuncture can down-regulate C/EBP homologous protein expression and up-regulate glucose-regulated protein 78 protein expression level in epilepsy rats, which may be the protective effect of acupuncture on epileptic brain injury (17). Another animal study suggests that the effect of acupuncture on epileptic rats may be related with change of GAD (67) mRNA level in dentate gyrus region (18). The thalamus plays a crucial role in the sensory transmission and is closely related to the epilepsy genesis. Since the thalamus is a converging structure of acupuncture and vagus nerve stimulation, and the activity of thalamic neurons can be modulated by acupuncture, this may be one of the mechanisms of acupuncture in treating epilepsy (19). In 2018, Deng et al. (20) conducted a systematic review and meta-analysis, which detected that acupuncture therapy might have a positive effect in the treatment of epilepsy. However, the evidence was insufficient and limited due to the high risk of bias and the low quality of the included studies. A retrospective matched cohort study has shown that stroke participants who received acupuncture had a lower chance of epilepsy than those who did not. Nevertheless, whether acupuncture can be used for primary and secondary prevention of PSE needs to be further validated in prospective cohort trials (21).

Current evidence on the effects of acupuncture on epilepsy in stroke patients is insufficient. Now, we will use acupuncture as a clinical TCM rehabilitation method for PSE. Therefore, we hypothesize that acupuncture will have better clinical efficacy than sham acupuncture in clinical symptoms, cognitive function, and quality of life.

\section{METHODS AND ANALYSIS}

\section{Study Design}

A multicentre randomized controlled trial (RCT) will be conducted at the Tianjin Nankai Hospital, Tianjin Rehabilitation and Convalescence Center of PLA, Tianjin Gong An Hospital, The Second Hospital Affiliated to Tianjin University of TCM, all of which are teaching hospitals. The protocol was registered with the China Clinical Trial Registry (item number: ChiCTR2100046114), and this study protocol has been approved by the Ethics Committee of Tianjin Gong An Hospital (item number: 2020003). A total of 120 participants will be recruited. All participants will provide written informed consent at the time of recruitment. PSE participants will be recruited and randomly assigned to either conventional therapy plus acupuncture or conventional therapy plus sham acupuncture. The evaluator will evaluate and analyze the results at four points (before treatment, 4 weeks, 8 weeks, and 1 year). Tianjin University of TCM will be responsible for data management and statistics. The flow chart of the trial is illustrated in Figure 1, and Table 1 shows the schedule of measurements.

\section{Participant Recruitment}

Epilepsy is diagnosed according to International League Against Epilepsy guidelines, and consequently, one unprovoked seizure occurring more than 1 week after stroke will be defined as PSE (22-24). Participants with a first post-episode of seizures a week after a stroke confirmed will be recruited. The PSE will be diagnosed following an examination, brain CT/MRI scan, and electroencephalogram (EEG) of participants. Recruitment advertisements will be posted on, the hospital websites, inpatient departments, WeChat, Facebook, Tik Tok, and other official platforms to recruit potential patients. The informed consent process will be conducted by the study coordinator or principal investigator, and if the participants agree to sign the informed consent, they will be screened to confirm that potential participants meet the eligibility criteria listed below.

\section{Inclusion Criteria}

Participants with the following conditions will be included: (1) patients with PSE; (2) age range from 18 to 75 years old; (3) in stable condition and conscious; (4) no psychiatric drugs have been taken in 2 months; (5) the informed consent is signed by the participant or his/her immediate family; (6) regardless of whether the patient taking anticoagulant and/or antiplatelet therapy (25). 


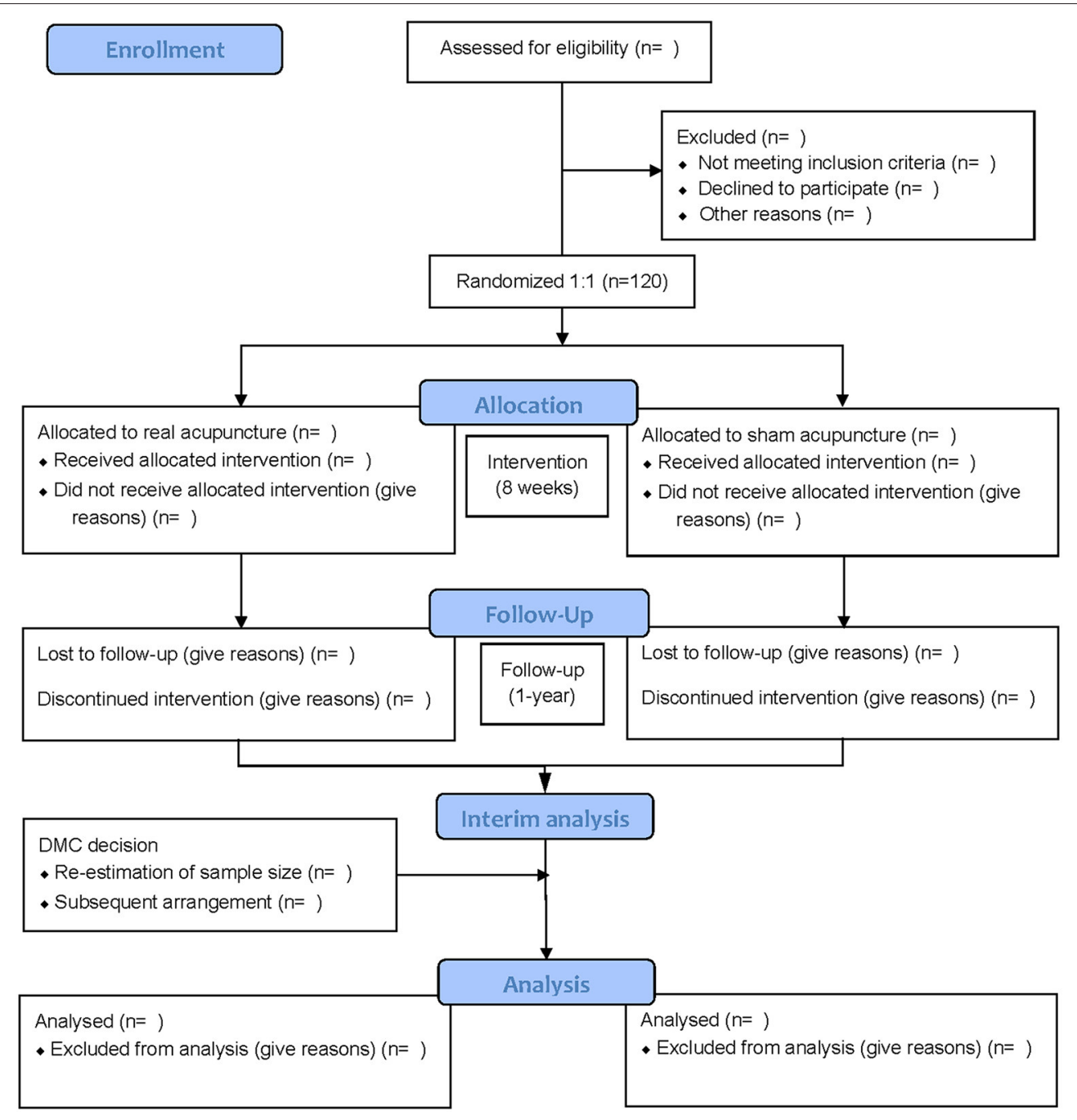

FIGURE 1 | Flow chart of the trial. DMC, data monitoring committee.

\section{Exclusion Criteria}

Participants with the following conditions will be excluded: (1) a history of psychiatric disorders; (2) being pregnant or lactating; (3) a personal or family history of epilepsy; (4) central nervous system infection, traumatic brain injury, or intracranial tumor; (5) alcoholism, poisoning, cortical dysplasia, or other condition that may cause seizures; (6) suffering from psychogenic nonepileptic seizure by video-EEG evaluation (26).

\section{Randomization and Blinding}

Randomization will be computer-generated by independent researchers using SAS 9.3 (SAS Institute Inc., Cary, NC, USA) software. Researchers will place the generated list of random numbers into sequentially numbered, opaque, sealed envelopes. Based on the information received from the envelope, consecutive participants will be randomly assigned in a 1:1 ratio to acupuncture group (AG) or sham acupuncture group (SAG). Acupuncturists will be appointed to perform the acupuncture for both groups separately. The research coordinator will not be involved in assessment or treatment. And they will notify the patients that they would be likely to receive either a "lesspainful acupuncture developed especially for this study" (sham) or a "traditional Chinese acupuncture" (true). All patients and statisticians will be blinded to group assignments.

\section{Interventions and Comparison}

Both groups will receive routine stroke care and treatment during the whole 8 -week study. The program was designed according to the Chinese Stroke Association Stroke Council Guideline will be consistent across groups (27). Chinese herbal medicine will be prohibited during the trial. The routine use of prophylactic anticonvulsant drugs for PSE remains largely controversial. Although many physicians use AEDs as secondary prevention of PSE, there is insufficient evidence on which AEDs are most effective in preventing recurrent epilepsy $(1,4,5)$. The 2013 International League Against Epilepsy 
TABLE 1 | Study schedule showing the time points for enrollment and assessment.

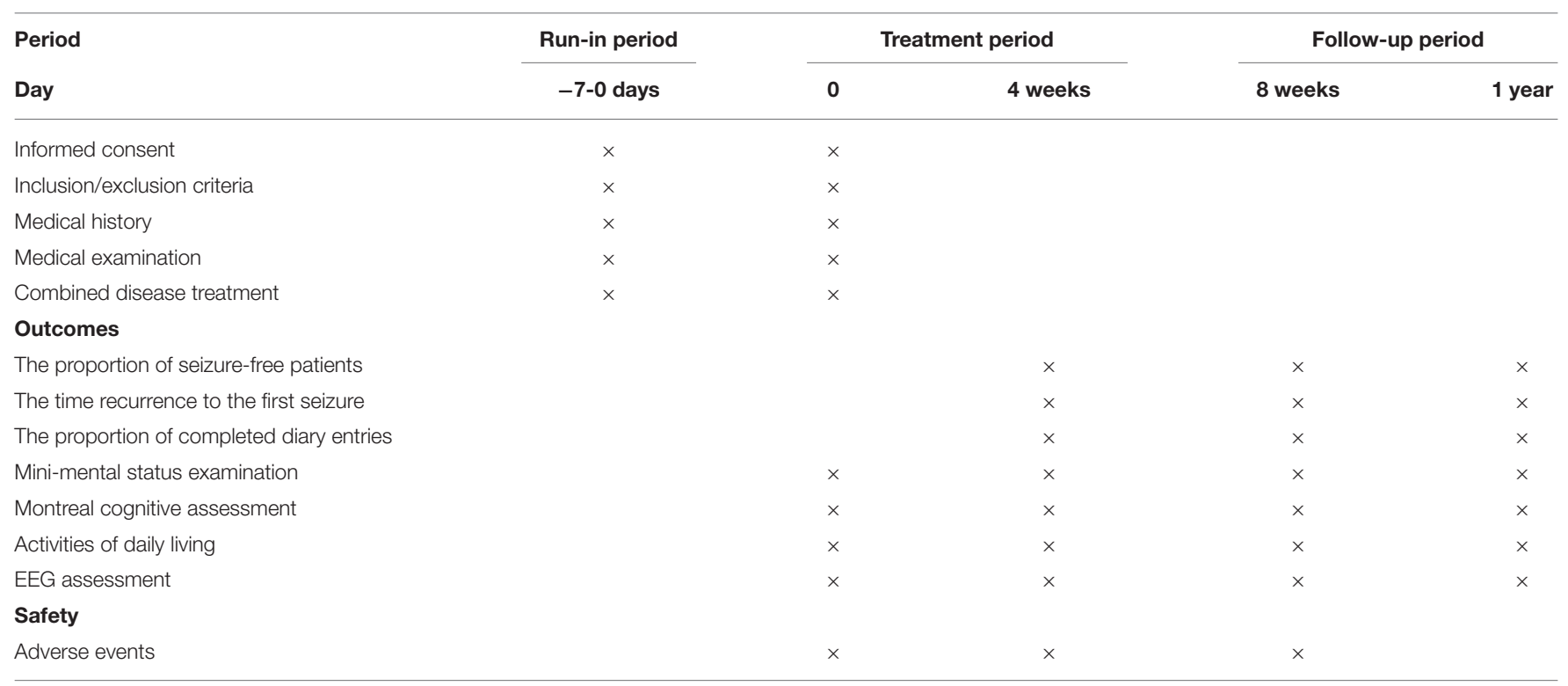

$\times$ represents the beginning of information collection.

(ILAE) report suggested carbamazepine (CBZ), Levetiracetam (LEV), phenytoin (PHT) and zonisamide (ZNS) have "level A" evidence (28). Multicenter prospective RCT suggests that LEV in monotherapy is a safe and effective therapeutic option in elderly patients who have suffered PSE (29). So, we will use AEDs to prevent PSE recurrence. AEDs should be tailored to patients according to their age, type of seizure, drug interaction, physical health and other factors. Medication adjustment is handled by neurologists with more than 5 years of clinical experience. If participants present repeated, uncontrolled seizures, they should be given rescue medications that rapidly control the seizure, such as diazepam, midazolam propofol and so on, along with effective supportive treatment, symptomatic treatment, such as keeping the respiratory tract open, correcting acid-base balance, correcting electrolyte disturbances, and preventing or controlling infection (30).

We plan to start acupuncture at the beginning of treatment (together with the AEDs). Acupuncture treatment for the assigned participants will be performed by acupuncturists who have at least 5 years of clinical experience. The participants will receive $30 \mathrm{~min}$ of real or sham acupuncture sham acupuncture in a supine position, three times a week for eight weeks. To improve and monitor compliance, those who complete all treatments and assessments will receive financial compensation. Besides, each treatment form and evaluation form will be filled out and signed by patients and investigators.

\section{Acupuncture Group}

After skin disinfection, disposable acupuncture needles (size $0.3 \times 40 \mathrm{~mm}$, Xin xinglin brand, manufactured by Beijing Tianyuheng technology company in Beijing, China) will be inserted with the hand. The choice of acupoints refers to the textbook of Chinese acupuncture and moxibustion (31). The body acupuncture points including RN15 (Jiuwei), PC5 (Jianshi), SI3 (Houxi), ST40 (Fenglong). The acupuncture areas of the two groups (AG and $\mathrm{SAG}$ ) are shown in Figure 2. The locations of the AG and SAG are detailed in Table 2. Acupoints will be stimulated manually (depth of insertion varies from 15 to $30 \mathrm{~mm}$ ) until patients feel soreness, distension or heaviness (the reaction of "De Qi"). "De Qi” has been shown to be vital for the differential neurophysiological analgesic mechanism between non-responders and responders to acupuncture (32).

\section{Sham Acupuncture Group}

Manipulations of sham acupuncture. The same stainless needles (size $0.3 \times 40 \mathrm{~mm}$, described above) will be used. Sham acupuncture in the study is defined as acupuncture with minimal stimulation at the non-meridian and non-acupoint areas $(1 \mathrm{~cm}$ lateral away from the actual acupoints) and superficial needle insertion (varies from 1 to $3 \mathrm{~mm}$ ). The number of insertion needles, the time of needle retention, and the frequency of treatment are the same as $\operatorname{AG}(33,34)$.

\section{Outcome Measures The Primary Outcome}

The primary endpoint will be defined as the appearance of a second seizure under treatment or by finishing the 12-month follow-up period without seizures $(29,35)$.

\section{Secondary Outcomes}

Secondary outcomes will include the time recurrence to the first seizure. An event is defined as the time of the first relapse of a seizure after baseline; the time of the seizure is calculated as the difference of weeks between the date of the visit identifying the crisis and the date of the baseline visit. The differences in cognitive functions and quality of life, which will be estimated using the Mini-Mental Status Examination 


\section{- Acupoints \\ - Non-acupoints}

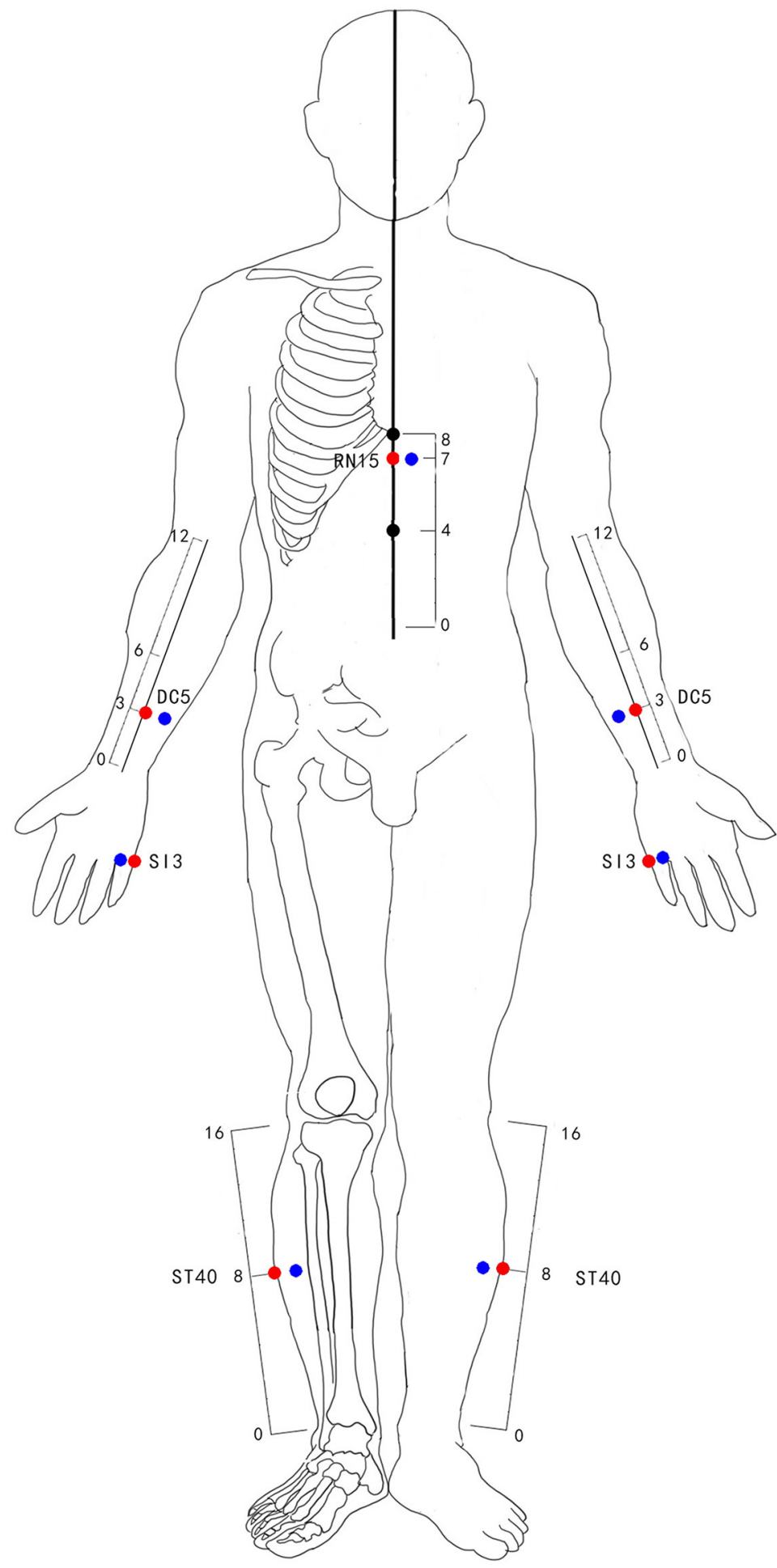

FIGURE 2 | The acupuncture areas of the acupuncture group and sham acupuncture group. 
TABLE 2 | Locations of real and sham acupuncture.

\begin{tabular}{lll}
\hline Points & Real acupuncture location & Sham acupuncture location \\
\hline RN15 (Jiuwei) & $\begin{array}{l}\text { On the upper abdomen, } 1 \text { cun inferior to the xiphisternal junction, on the } \\
\text { anterior median line. } \\
\text { On the anterior aspect of the forearm, between the tendons of the palmaris } \\
\text { longus and the flexor carpi radialis, } 3 \text { cun proximal to the palmar wrist } \\
\text { crease. } \\
\text { On the dorsum of the hand, in the depression proximal to the ulnar side of } \\
\text { the fifth metacarpophalangeal joint, at the border between the red and white } \\
\text { fl3(Houxi) } \\
\text { flesh. }\end{array}$ & $\begin{array}{l}1 \mathrm{~cm} \text { lateral away from the actual } \\
\text { points of body acupuncture. }\end{array}$ \\
On the anterolateral aspect of the leg, lateral border of the tibialis anterior \\
muscle, 8 cun superior to the prominence of the lateral malleolus.
\end{tabular}

(36), Montreal Cognitive Assessment (37), the Activities of daily living (ADL) (38) and EEG assessment. EEG examinations will be performed at the beginning of the study, during the treatment and follow-up period. Assessments will be conducted by a researcher, who is unaware of the treatment to which the participant is assigned. To evaluate compliance with treatment, the proportion of completed diary entries over the 8-weeks research period will be calculated.

The outcomes will be evaluated at weeks $0,4,8$, and 12 months. To assess the long-term prognosis, we will follow up with all patients 1 year later through clinic visits, home visits, social media, or telephone contact to collect data on the time and frequency of their occurrence.

\section{Incidence of Adverse Events}

The patients will be requested to freewill report any acupuncturerelated adverse event (AEs). AEs will be recorded and evaluated by the researchers. Acupuncture-related AEs may include bleeding, local infection, soreness, sweating, and so on.

\section{Data Collection and Management}

We will gather basic information, disease severity assessment, and chemical examinations. The investigator will fill in the data separately in the case report forms (CRFs) and research medical record. Based on the need for data traceability, the original information will be saved in the study of medical records. CRF is designed to convenient for data entry input. Participants are identified by a code and their personal information is not displayed.

\section{Statistical Analysis}

The researchers of Tianjin University of TCM will perform statistical analysis, and they will analyze the data using SAS 9.3 software. Statistical analysis is conducted by researchers with statistical qualifications. Results data will be analyzed in accordance with the intent-to-treat principle, including all patients after baseline assessment, regardless of whether they received treatment or not. Statisticians will use descriptive statistics to summarize the demographic characteristics of the two groups. We will describe continuous variables in terms of mean and standard deviation, and use the $t$-test. Categorical variables will be undertaken using the $\chi 2$ test.

\section{DISCUSSION}

Stroke is one of the major global health problems, and its disease burden continues to increase due to the increasing aging of the population. PSE is a common complication after stroke, and although many doctors prescribe AEDs as secondary prevention of PSE, evidence on the efficacy and safety of the drugs is still limited (39). Given the lack of clear evidence of drugs for PSE, complementary and alternative medicine may have a promising future in the treatment of PSE from this perspective. Acupuncture is a simple, effective, and safe complementary and alternative therapy for various diseases, including stroke and epilepsy (11, 40-45). Based on published systematic reviews, acupuncture seems to be a promising therapy for epilepsy (20, 46). However, few RCTs acupuncture treatment for PSE has been published.

We have carefully designed the current RCT to provide reliable evidence of the advantages and effects of acupuncture on PSE. Computer-generated randomization of numbers and allocation concealment will be used to minimize selection bias. The evaluators will be blinded to the group allocation. In addition, we set up a sham acupuncture group to solve the problem of the blinding method. In summary, we will conduct an RCT to test the efficacy of acupuncture in 120 patients diagnosed with PSE. We plan to publish our data in a high-profile emergency medicine or neurology journal. It certainly largely depends on the results of the research. We will do our best to discharge our administrative duties.

\section{TRIAL STATUS}

This protocol version is number 4.0, dated 1 May 2021. The first participant will start on December 2022; the recruitment end date is expected to be 31 December 2027.

\section{ETHICS STATEMENT}

This study was ratified by the ethics committee of Tianjin Gong An Hospital (item number: 2020003). The results will be disseminated in China and at international conferences, and published in an international peer-reviewed journal. 


\section{AUTHOR CONTRIBUTIONS}

$\mathrm{KZ}$ and $\mathrm{ZZ}$ carried out the studies and drafted the manuscript. YZ, MZ, and BZ revised the manuscript. All authors have read and approved this version of the manuscript.

\section{REFERENCES}

1. Feyissa AM, Hasan TF, Meschia JF. Stroke-related epilepsy. Eur J Neurol. (2019) 26:18-e3. doi: 10.1111/ene.13813

2. Reddy DS, Bhimani A, Kuruba R, Park MJ, Sohrabji F. Prospects of modeling poststroke epileptogenesis. J Neurosci Res. (2017) 95:1000-16. doi: 10.1002/jnr.23836

3. Gasparini S, Ascoli M, Brigo F, Cianci V, Branca D, Arcudi L, et al. Younger age at stroke onset but not thrombolytic treatment predicts poststroke epilepsy: an updated meta-analysis. Epilepsy Behav. (2020) 104:106540. doi: 10.1016/j.yebeh.2019.106540

4. Holtkamp M, Beghi E, Benninger F, Kälviäinen R, Rocamora R, Christensen $\mathrm{H}$ et al. European stroke organisation guidelines for the management of post-stroke seizures and epilepsy. Eur Stroke J. (2017) 2:103-15. doi: $10.1177 / 2396987317705536$

5. Galovic M, Ferreira-Atuesta C, Abraira L, Döhler N, Sinka L, Brigo F, et al. Seizures and epilepsy after stroke: epidemiology, biomarkers and management. Drugs Aging. (2021) 38:285-99. doi: 10.1007/s40266-021-00837-7

6. Zhuang Y, Xing JJ, Li J, Zeng BY, Liang FR. History of acupuncture research. Int Rev Neurobiol. (2013) 111:1-23. doi: 10.1016/B978-0-12-411545-3.00001-8

7. He Y, Guo X, May BH, Zhang AL, Liu Y, Lu C, et al. Clinical evidence for association of acupuncture and acupressure with improved cancer pain: a systematic review and meta-analysis. JAMA Oncol. (2020) 6:271-8. doi: 10.1001/jamaoncol.2019.5233

8. Tang Q, Zhang K. Association of acupuncture and acupressure with improved cancer pain. JAMA Oncol. (2020) 6:938. doi: 10.1001/jamaoncol.2020.0616

9. Zhang K, Gao C, Li C, Li Y, Wang S, Tang Q, et al. Acupuncture for acute pancreatitis: a systematic review and meta-analysis. Pancreas. (2019) 48:1136-47. doi: 10.1097/MPA.0000000000001399

10. Zhang K, Li C, Gao C, Zhao C, Tang Q, Zhai J, et al. Efficacy and safety of acupuncture as an adjuvant treatment for acute pancreatitis: a protocol of systematic review and meta-analysis. BMJ Open. (2019) 9:e029327. doi: 10.1136/bmjopen-2019-029327

11. Zhang K, Tang Q, Zhao C. Traditional manual acupuncture combined with rehabilitation therapy for shoulder hand syndrome after stroke within the Chinese healthcare system. Clin Rehabil. (2019) 33:1699-700. doi: 10.1177/0269215519877739

12. Han Z, Wang J, Zhang K, Tang Q. The ethics of COVID-19 clinical trials: new considerations in a controversial area. Integr Med Res. (2020) 9:100425. doi: 10.1016/j.imr.2020.100425

13. Han Z, Zhang Y, Wang P, Tang Q, Zhang K. Is acupuncture effective in the treatment of COVID-19 related symptoms? Based on bioinformatics/network topology strategy. Brief Bioinform. (2021) 2021:bbab110. doi: 10.1093/bib/bbab110

14. Cheng FK. The use of acupuncture in patients with Parkinson's disease. Geriatr Nurs. (2017) 38:302-14. doi: 10.1016/j.gerinurse.2016.11.010

15. Maa E, Applegate M, Keniston A. Auricular acupuncture for the treatment of nonepileptic seizures: a pilot study. Epilepsy Behav. (2020) 111:107329. doi: 10.1016/j.yebeh.2020.107329

16. Li QQ, Shi GX, Xu Q, Wang J, Liu CZ, Wang LP. Acupuncture effect and central autonomic regulation. Evid Based Complement Alternat Med. (2013) 2013:267959. doi: 10.1155/2013/267959

17. Yang F, Ma Y, Ang WP, Chen H, Du WD, Wu SB, et al. Effects of acupuncture intervention on expression of glucose-regulated protein 78 and C/EBP homologous protein in hippocampal CA 1 region in rats with hyperspasmia. Zhen Ci Yan Jiu. (2014) 39:267-71.

18. Guo J, Liu J, Fu W, Ma W, Xu Z, Yuan M, et al. The effect of electroacupuncture on spontaneous recurrent seizure and expression of GAD

\section{FUNDING}

This study was funded by the rerional
Natural Science Foundation of China
number 81774056).

(67) mRNA in dentate gyrus in a rat model of epilepsy. Brain Res. (2008) 1188:165-72. doi: 10.1016/j.brainres.2007.10.017

19. Chen S, Wang S, Rong P, Liu J, Zhang H, Zhang J. Acupuncture for refractory epilepsy: role of thalamus. Evid Based Complement Alternat Med. (2014) 2014:950631. doi: 10.1155/2014/950631

20. Deng BW, Luo XZ, Tang CZ, Zhang X. A meta-analysis of the effectiveness of acupuncture in the treatment of epilepsy. Zhen Ci Yan Jiu. (2018) 43:263-8. doi: 10.13702/j.1000-0607.170347

21. Weng SW, Liao CC, Yeh CC, Chen TL, Lane HL, Lin JG, et al. Risk of epilepsy in stroke patients receiving acupuncture treatment: a nationwide retrospective matched-cohort study. BMJ Open. (2016) 6:e010539. doi: 10.1136/bmjopen-2015-010539

22. Zelano J, Holtkamp M, Agarwal N, Lattanzi S, Trinka E, Brigo F. How to diagnose and treat post-stroke seizures and epilepsy. Epileptic Disord. (2020) 22:252-63. doi: 10.1684/epd.2020.1159

23. Hassani M, Cooray G, Sveinsson O, Cooray C. Post-stroke epilepsy in an ischemic stroke cohort-incidence and diagnosis. Acta Neurol Scand. (2020) 141:141-7. doi: 10.1111/ane.13174

24. Napon C, Dabilgou A, Kyelem J, Kaboré J. Post-stroke epilepsy in Burkina Faso (West Africa). J Neurol Sci. (2016) 368:47-8. doi: 10.1016/j.jns.2016.06.038

25. Lee M, Lee S, Kim E, Cho YE, Kang JW, Lee JD. Evaluation of bleeding-related adverse events following acupuncture treatment in patients on anticoagulant or antiplatelet drugs: a prospective observational study. Complement Ther Med. (2018) 41:23-8. doi: 10.1016/j.ctim.2018.08.006

26. Anzellotti F, Dono F, Evangelista G, Di Pietro M, Carrarini C, Russo $\mathrm{M}$, et al. Psychogenic non-epileptic seizures and pseudo-refractory epilepsy, a management challenge. Front Neurol. (2020) 11:461. doi: 10.3389/fneur.2020.00461

27. Liu L, Chen W, Zhou H, Duan W, Li S, Huo X, et al. Chinese Stroke Association guidelines for clinical management of cerebrovascular disorders: executive summary and 2019 update of clinical management of ischaemic cerebrovascular diseases. Stroke Vasc Neurol. (2020) 5:159-76. doi: 10.1136/svn-2020-000378

28. Glauser T, Ben-Menachem E, Bourgeois B, Cnaan A, Guerreiro C, Kälviäinen $\mathrm{R}$, et al. Updated ILAE evidence review of antiepileptic drug efficacy and effectiveness as initial monotherapy for epileptic seizures and syndromes. Epilepsia. (2013) 54:551-63. doi: 10.1111/epi.12074

29. Consoli D, Bosco D, Postorino P, Galati F, Plastino M, Perticoni GF, et al. Levetiracetam versus carbamazepine in patients with late poststroke seizures: a multicenter prospective randomized open-label study (EpIC Project). Cerebrovasc Dis. (2012) 34:282-9. doi: 10.1159/000342669

30. Bank AM, Bazil CW. Emergency management of epilepsy and seizures. Semin Neurol. (2019) 39:73-81. doi: 10.1055/s-0038-1677008

31. Qiu ML, Zhang SS, Yu ZQ. Chinese Acupuncture and Moxibustion Science. Shanghai: Shanghai Science and Technology Press (2018).

32. Asghar AU, Green G, Lythgoe MF, Lewith G, MacPherson H. Acupuncture needling sensation: the neural correlates of deqi using fMRI. Brain Res. (2010) 1315:111-8. doi: 10.1016/j.brainres.2009.12.019

33. Chen L, Fang J, Jin X, Keeler CL, Gao H, Fang Z, Chen Q. Acupuncture treatment for ischaemic stroke in young adults: protocol for a randomised, sham-controlled clinical trial. BMJ Open. (2016) 6:e010073. doi: 10.1136/bmjopen-2015-010073

34. Tan JY, Suen LK, Wang T, Molassiotis A. Sham acupressure controls used in randomized controlled trials: a systematic review and critique. PLOS ONE. (2015) 10:e0132989. doi: 10.1371/journal.pone.0132989

35. Gilad R, Sadeh M, Rapoport A, Dabby R, Boaz M, Lampl Y. Monotherapy of lamotrigine versus carbamazepine in patients with poststroke seizure. Clin Neuropharmacol. (2007) 30:189-95. doi: 10.1097/WNF.0b013e3180333069 
36. Grut M, Fratiglioni L, Viitanen M, Winblad B. Accuracy of the minimental status examination as a screening test for dementia in a swedish elderly population. Acta Neurol Scand. (1993) 87:312-7. doi: 10.1111/j.1600-0404.1993.tb05514.x

37. Nasreddine ZS, Phillips NA, Bédirian V, Charbonneau S, Whitehead V, Collin I, et al. The Montreal Cognitive Assessment, MoCA: a brief screening tool for mild cognitive impairment. J Am Geriatr Soc. (2005) 53:695-9. doi: 10.1111/j.1532-5415.2005.53221.x

38. Mlinac ME, Feng MC. Assessment of activities of daily living, selfcare, and independence. Arch Clin Neuropsychol. (2016) 31:506-16. doi: 10.1093/arclin/acw049

39. Tanaka T, Ihara M. Post-stroke epilepsy. Neurochem Int. (2017) 107:219-28. doi: 10.1016/j.neuint.2017.02.002

40. Zhang K, Li Y, Tang Q. Acupuncture for stable angina pectoris: a few noteworthy additions. Eur J Prev Cardiol. (2020) 27:2063-4. doi: $10.1177 / 2047487319889721$

41. Zhang K, Tang Q. Acupuncture on aromatase inhibitor-induced arthralgia in patients with breast cancer. Breast. (2019) 45:119. doi: 10.1016/j.breast.2019.01.010

42. Tang Q, Zhang K. Is acupuncture effective for knee osteoarthritis? Comment on a recent trial. Clin Rehabil. (2019) 33:1697-8. doi: 10.1177/0269215519863554

43. Zhang K, Tang QL, Gao C. Non-pharmacologic treatments for symptoms of diabetic peripheral neuropathy: a systematic review - methodological issues are a matter for concern. Curr Med Res Opin. (2019) 35:1319-20. doi: 10.1080/03007995.2019.15 98135
44. Zhang K, Li Y, Tang Q. Acupuncture for breathlessness in advanced diseases: methodological issues. J Pain Symptom Manage. (2020) 59:e3-4. doi: 10.1016/j.jpainsymman.2019.11.020

45. Zhang K. Birth preparation acupuncture for normalising birth: a few noteworthy additions. J Obstet Gynaecol. (2021) 41:166. doi: 10.1080/01443615.2020.1785411

46. Cheuk DK, Wong V. Acupuncture for epilepsy. Cochrane Database Syst Rev. (2014) CD005062. doi: 10.1002/14651858.CD005062.pub4

Conflict of Interest: The authors declare that the research was conducted in the absence of any commercial or financial relationships that could be construed as a potential conflict of interest.

Publisher's Note: All claims expressed in this article are solely those of the authors and do not necessarily represent those of their affiliated organizations, or those of the publisher, the editors and the reviewers. Any product that may be evaluated in this article, or claim that may be made by its manufacturer, is not guaranteed or endorsed by the publisher.

Copyright (c) 2021 Zhang, Zhao, Zhang, Zhang and Zhou. This is an open-access article distributed under the terms of the Creative Commons Attribution License (CC $B Y)$. The use, distribution or reproduction in other forums is permitted, provided the original author(s) and the copyright owner(s) are credited and that the original publication in this journal is cited, in accordance with accepted academic practice. No use, distribution or reproduction is permitted which does not comply with these terms. 